\title{
Advancing Nuclear Medicine in Australia over 3 Decades
}

\author{
A Conversation Between Rodney Hicks, Johannes Czernin, and Ken Herrmann
}

\author{
Rodney Hicks ${ }^{1}$, Johannes Czernin ${ }^{2}$, and Ken Herrmann ${ }^{3}$ \\ ${ }^{1}$ Peter MacCallum Cancer Institute, Melbourne, Australia; ${ }^{2}$ David Geffen School of Medicine at UCLA, Los Angeles, California; and \\ ${ }^{3}$ Universitätsklinikum Essen, Essen, Germany
}

$\mathbf{J}$ ohannes Czernin, editor-in-chief of The Journal of Nuclear Medicine, and Ken Herrmann, a professor of nuclear medicine at the Universitätsklinikum Essen (Germany), talked with Rodney Hicks, a professor of medicine and radiology at the University of Melbourne, head of the Molecular Imaging and Targeted Therapeutic Laboratory at the Sir Peter MacCallum Department of Oncology, and cochair of the Neuroendocrine Service at the Peter MacCallum Cancer Centre (Melbourne, Australia). Dr. Hicks is recognized as a pioneer in PET assessment of cancer and continues to be an international leader in clinical trials and implementation of novel PET tracers and theranostic pairs. The PET program he founded in Australia included only the third such scanner in the country. In 2019, the unit passed the milestone of 100,000 scans. Today, the Hicks laboratory, as part of the Centre for Cancer Imaging, uses genomic approaches and in vivo imaging of tumor biology in models of human cancer, with remarkable accomplishments in neuroendocrine tumor (NET) imaging and treatments. In 2018, his group was the first outside Europe to be recognized as a Centre of Excellence in the European Neuroendocrine Tumor Society Network.

Dr. Hicks holds numerous national and international research grants and is editor-in-chief of Cancer Imaging, as well as serving on multiple editorial boards. He has authored more than 500 peerreviewed articles. In 2015, he was inducted as a Fellow of the Australian Academy of Health and Medical Sciences. In 2019 he received the gold medal from the International Cancer Imaging Society in recognition of his exceptional contribution to oncologic imaging and international education.

Dr. Czernin: There is an interesting parallel between us that I had almost forgotten, and that is that we both started out in nuclear cardiology. I was fortunate to be trained and mentored by Heinrich Schelbert. Markus Schwaiger served as your mentor at Ann Arbor, MI. Can you tell us a little bit about how you got to Michigan from Australia and why you later focused on oncology?

Dr. Hicks: I thought exactly the same thing - that our roots are so similar and how lucky we've both been to have come in contact with absolute pioneers in PET. As you know, PET really started with the idea of looking at the brain and the heart. Oncology came in as a poor third place in people's thinking, partly because there was no whole-body reconstruction. When I saw the first wholebody image reconstruction come out of the UCLA in a paper writ-

COPYRIGHT (C) 2022 by the Society of Nuclear Medicine and Molecular Imaging DOI: 10.2967/jnumed.121.263338 ten by Magnus Dahlbom, I knew that PET was going to be the future of oncologic imaging. This changed my career direction. I had seen the work that Richard Wahl did in the very early days on breast cancer. On the first oncologic PET scan I ever saw, we identified an internal mammary and axillary node and a bone metastasis that were completely occult on CT. So my career direction changed from being a noninvasive car-

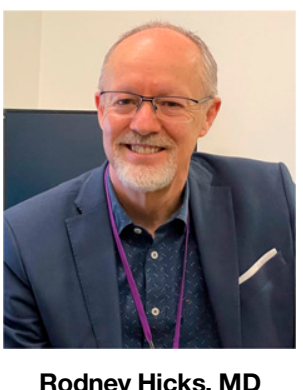
diologist doing echocardiography, cardiac CT, MRI, and nuclear cardiology to being fascinated by oncology.

Dr. Czernin: I saw your paper from 1993 in the American Journal of Cardiology (1993;71:529-535) on ${ }^{11}$ C-acetate and measuring oxygen use of the heart in patients with aortic stenosis. You were already involved early on in cardiac molecular imaging.

Dr. Hicks: This was certainly Markus' influence. A lot of what I learned from nuclear cardiology directly translated into my thinking about oncology: the concept that you could look at more than one aspect of biology with tracers that were quantitatively and qualitatively evaluable on PET or the idea to combine perfusion and metabolic imaging to identify different myocardial cell populations, including necrosis, hibernation, ischemia, and normal tissue. For me, this became the model for nuclear oncology. ${ }^{18} \mathrm{~F}-\mathrm{FDG}$ is extremely good at identifying sites of disease, by informing us about glycolytic tissue activity, an important hallmark but not the only aspect of tumors. We subsequently added hypoxia imaging at Peter Mac to look at how hypoxia impacts prognosis. The idea that 2 tracers could be used to identify subpopulations of cells that coexisted in the same tumor became really important. We've extended that into conceptual thinking about tumor heterogeneity. For instance, NETs can have populations that express somatostatin receptors and are highly glycolytic, whereas others have low receptor expression and high glycolysis and still others have high receptor expression and low glycolysis. They all behave in different ways. They have different available therapeutic options and certainly different prognoses. Similar phenotyping by imaging can now be done in many cancers, including, of course, prostate cancer.

Dr. Herrmann: Let us get back for a moment to your career switch from cardiology to oncology.

Dr. Hicks: After returning to Australia from Michigan I worked at the Repatriation General Hospital in Adelaide in a dual role, with coronary care 1 week per month and as director of nuclear cardiology in the nuclear medicine department. Our pay was really 
low, so we were allowed to have 1 day per week where we could go and earn a real living. A friend of mine at Peter Mac invited me to go along in the early 1990s, just after I got back from Michigan. There, I became really fascinated by the opportunities in oncology. This also coincided with the outbreak of the AIDS epidemic in Australia. We served many patients who had Kaposi sarcoma and lymphomas. We became involved in dual-tracer SPECT phenotyping studies that helped to arrive at correct diagnoses. This again got me very interested in the pluralistic nature of nuclear medicine and the concept of using imaging for phenotyping.

Dr. Czernin: Were you actually trained in radiology?

Dr. Hicks: No, I was trained in internal medicine. Although I had limited experience in oncology at the time, nuclear medicine at Peter Mac was very poorly equipped. They needed someone to bring it into the modern era of molecular imaging. It was staffed by a couple of radiologists who were nearing retirement, and they mostly performed bone scans. We eventually ended up changing from planar to SPECT and started doing some interesting work. From a very early stage, I was educating the oncologists in the use of molecular imaging to characterize cancers, particularly using ${ }^{67} \mathrm{Ga}$-citrate in lymphoma and melanoma and ${ }^{201} \mathrm{Tl}$ in sarcoma. So, when PET became available, we were early adopters. In 1996, we started our PET program - it is 25 years old in 2021.

Dr. Herrmann: What was the size of your department at that time?

Dr. Hicks: In 1996, I was the only nuclear medicine physician in the department. We had 2 full-time technologists working in the PET section downstairs and 2 full-timers and a part-timer upstairs in the nuclear medicine department. In the PET facility, one of the
Dr. Herrmann: You have a big operation now. We want to make sure that our readers understand that you took a very small group and converted it into something big.

Dr. Hicks: We have 6 or 7 nuclear medicine attendings, 3 fellows, 2 radiopharmacists, and 3 radiochemists, so it's a moderatesized program. We have 30-40 technologists and run a very busy theranostic program.

Dr. Czernin: How many patients are you treating every day?

Dr. Hicks: In the pre-COVID era, our therapeutic nuclear medicine program was much busier than it is at present. We were delivering close to 700 treatments per year, primarily to patients with NETs. In recent years, we have seen growth in prostate cancer treatment using ${ }^{177} \mathrm{Lu}$-prostate-specific membrane antigen $\left({ }^{177} \mathrm{Lu}\right.$-PSMA), primarily in a clinical trial setting. Recently we have been treating about 8 neuroendocrine patients and about 10 prostate patients per week. Our prostate cancer program has gone kind of nuts - almost threatens to overwhelm the theranostic program.

Dr. Herrmann: It's a pretty decent number.

Dr. Hicks: It's a busy program. It keeps us on our toes, especially since we are very actively involved in obtaining patient consent, checking laboratory results, delivering therapy, and then having consultations with patients after posttreatment scanning to discuss further management or follow-up plans. We also do many consultations for patients either being considered for peptidereceptor radionuclide therapy (PRRT) or in surveillance programs. As well as NET and prostate cancer, we treat pheochromocytoma and paraganglioma, Merkel cell carcinoma, thyroid cancer, and children with neuroblastoma. I like to think that we have the most

\section{"I like to think that we have the most interesting oncologic practice in the building and act as what}

I call 'theranosticians.

technologists did nuclear cardiology on the other side of the corridor from the PET scanner. At the time, we had no funding and I had quite a profitable private practice in nuclear cardiology, so I made money on one side of the corridor, which my CEO (who was a nuclear physicist and quite an enlightened man) allowed me to spend to fund PET on the other side of the corridor. From a very early stage, then, we did PET at no charge to our patients.

Dr. Herrmann: Did you have a radiopharmacy? How did this compare to the size of the team now?

Dr. Hicks: Initially we purchased ${ }^{18}$ F-FDG from the Austin Hospital. The federal government set up 2 PET scanners, one in Sydney and one in Melbourne. The unit in Melbourne at the Austin Hospital was primarily a research facility to study the brain. They became increasingly interested in oncology, as Andrew Scott had come back from Memorial Sloan Kettering and was clearly very interested in oncologic PET. Soon it became clear that we needed our own supply, particularly since I was interested in developing radiotracers to complement ${ }^{18} \mathrm{~F}$-FDG. We purchased a cyclotron that had been purchased by Emory University but couldn't then be installed because the allocated space was co-opted as an MRI facility used for scanning athletes injured during the Olympic Games held in Atlanta in 1996. We set up solid targetry to make ${ }^{64} \mathrm{Cu},{ }^{86} \mathrm{Y}$, and ${ }^{124} \mathrm{I}$. I had a wonderful radiopharmacist, Peter Eu, and recruited a radiochemist, Peter Roselt, and got to play with some of these really cool longer-lived radioisotopes. We were very fortunate to get a couple of large government grants to develop novel radiopharmaceuticals. interesting oncologic practice in the building and act as what I call "theranosticians."

A key to our program has been the integration of PET into our theranostic selection and monitoring. We introduced ${ }^{68} \mathrm{Ga}$-DOTAoctreotate into Australia in 2009 and ${ }^{68}$ Ga-PSMA-11 in 2014. Coincidentally, we started our PRRT program in the same week in 1996 as that in which we did our first PET scan at Peter Mac. We're celebrating the 25 th anniversary of both programs. Our first PRRT patient was treated with a high administered activity with ${ }^{111}$ In-octreotide using peptide and a protocol kindly supplied by Eric Krenning of the Erasmus Medical Center in Rotterdam (The Netherlands). That patient was actually my cardiac stress nurse and had a fantastic response in several of her deposits, although one continued to grow. I thought this might have been a different pathology and so organized a PET scan. This turned out to be the only ${ }^{18}$ F-FDG-avid lesion and had demonstrated no uptake on her initial SPECT study. When we biopsied it, we found that it was a high-grade NET, and, unfortunately, she eventually died of that high-grade tumor. We used ${ }^{111}$ In-octreotide up until 2005 and combined it with chemotherapy after 1999. Although innovative at the time, it was very natural for our oncologists to consider combining radionuclide therapy with radiosensitizing chemotherapy, because they were doing it with external-beam radiotherapy. We were also involved in early trials with ${ }^{90} \mathrm{Y}$-DOTATOC and continued to use this for patients with larger tumor burdens until recently, even though we transitioned for the most part to ${ }^{177} \mathrm{Lu}$-DOTA-octreotate from 2005 onward. I still think that there is a place for ${ }^{90} \mathrm{Y}$ - and 
Auger electron-based radioligand therapy. The challenge we face now is that the nuclear medicine world seems to feel that the answer to all theranostic applications is ${ }^{177} \mathrm{Lu}$. It is my view that a strength of our specialty is that we have a panoply of isotopes with different half-lives and different physical energies, and we need to start to optimize how we combine these with the kinetics of uptake and retention of our targeting moieties.

Dr. Czernin: Optimization requires industry support. For example, ${ }^{225}$ Ac-PSMA will require a phase 3 trial, like the VISION trial. The same is true for ${ }^{225}$ Ac-DOTATATE trials that are coming up for NETs. My question is always: with all the different theranostic pairs emerging for various diseases, how is the supply problem going to be solved?

Dr. Hicks: We talk about personalized medicine, and yet we embrace these one-size-fits-all protocols in a regulatory sense. To me, this is absolutely nuts. As indicated earlier, I think we should use ${ }^{90} \mathrm{Y}$ for large lesions where responses to ${ }^{177} \mathrm{Lu}$-DOTATATE are not good. We achieved almost the best responses we'd ever seen in NETs when starting with yttrium and moving to lutetium. What we don't deal with is the small-volume stuff that's below the level of our detectability, and that's where the $\alpha$-emitters or Auger electrons come in. How are we ever going to design a clinical trial that cycles a patient through yttrium, lutetium, or a combination of lutetium plus actinium to hit both large and small lesions? Such trials are not doable. No industry partner is going to fund them. I believe that there needs to be a combination of centers that supply standardized protocols and also support for academic centers of excellence to provide more personalized treatments for complex cases, perhaps on the compassionate-use model used in Germany and Australia.

Dr. Herrmann: I would like to get your opinion on the supply problem. Do you think that ${ }^{177} \mathrm{Lu}$ can become a commodity within 2 or 3 years, or will this remain a critical issue as you're experiencing right now? Which other radionuclides will be commodities and which will still be limited in the next 5 years?

Dr. Hicks: One issue is regulatory, whether agencies are going to demand no-carrier-added lutetium. The no-carrier-added version increases cost and decreases availability. But, in my view, if the demand is there, money will be available to produce it. There will be an industrial solution that includes other agents or pairs, such as ${ }^{64 / 67} \mathrm{Cu}$, where ${ }^{67} \mathrm{Cu}$ can be made on a linear accelerator. Again, it's not very widely available yet, but industry will respond.

Dr. Herrmann: What do you think the role of dosimetry will be 5 years from now? How do we get there?

Dr. Hicks: It's like a bell-shaped curve. What we give as a standard administered activity is what people have learned is generally effective and generally safe. But this deals with only 2 SDs of the population in whom we're getting reasonable dose delivery to tumor with what I think is probably an excessive safety margin. Long-term follow-up studies suggest that we are delivering a very safe therapy. If medical oncologists were as worried about safety as we are, they would have almost no therapies, because their treatments tend to be much more toxic.

Dr. Czernin: But oncologists do individualize patient doses and would never accept the concept of one dose that fits all. That's why I think dosimetry will be very important. We are budgeting now for 2 or 3 dosimetrists to be hired over the next 4 or 5 years.

Dr. Hicks: I agree that there are a lot of inconsistencies in the ways oncologists judge our toxicity compared with their own, but we can take their lead and adapt our treatment to the individual circumstances of our patients. For example, there are clearly patients with large tumor burdens to whom we can give a lot more activity quite safely because of the tumor sink effect, with little collateral damage to normal tissues. At the other end of the spectrum are patients with impaired renal function, low bone marrow reserves, or a small tumor burden, in whom the standard administered activity may be too toxic. Perhaps on current protocols they would be excluded from treatment, but reduced administered activity might still be very effective. These 2 groups of patients, I think, are the ones in whom dosimetry should be mandatory and ideally prospective, not based on measuring the dose delivered after treatment, which is dose verification rather than dosimetry.

Dr. Herrmann: I want to pick your brain: What would kidney dose limits be? Would it still be 23 Gy as proposed by the U.S. Food and Drug Administration?

Dr. Hicks: Way above 23 Gy. We have been collecting posttreatment, quantitative SPECT/CT data for almost a decade. We have very long-term follow-up data on a large group of patients in whom we've measured the amount of delivered radiation. We know that we sometimes get in excess of 30 or 40 Gy to the kidneys without any significant long-term damage. We've been retreating patients with PRRT, some up to 20 cycles over a 14-year period, and many still have normal renal function, although some show damage to the kidneys. The main risk factor, in my opinion, is renal cortical thickness. Hydronephrotic kidneys, even if stented, seem particularly at risk because the residual nephron mass comes into closer proximity to the proximal convoluted tubules.

Dr. Czernin: The other issue that is often forgotten is that it depends on the population at which we are looking. For the VISION trial population, for example, I see no reason to be overly conservative with administrated activity. For such treatment in patients with hormone-sensitive disease, it might be a different story.

Dr. Hicks: The basis of the 23-Gy threshold was to keep the risk of dialysis at 5 years posttherapy at $5 \%$. Five years is a long way off for most patients with castrate-resistant metastatic prostate cancer but, as you note, may be an issue if treatment is moved earlier in the disease evolution.

Dr. Herrmann: And this 23-Gy limit was related to externalbeam radiation.

Dr. Hicks: Exactly, this was for external-beam radiation. If we put it in the context that few patients with uncontrolled progressive disease will live for 5 years and that they have an almost $100 \%$ chance of dying in that interval if disease is not controlled, then the imbalance in risk evaluation by regulators is evident. We have become so risk-averse that we don't see the clear and present danger that cancer poses to these patients.

Dr. Herrmann: How would you use dosimetry information in a prospective dosimetry trial?

Dr. Hicks: We have to collect the data to create the evidence for using dosimetry. But it is my experience that with sequential cycles of treatment, tumor dose decreases, the dose to normal organs increases, and thus the therapeutic index narrows with additional cycles. If that is the case, then isn't it logical to increase the administered activity for the early cycles when the therapeutic index is highest to try and achieve the greatest radiation delivery? The idea that NETs or prostate cancers don't have a dose-response curve is the most unscientific claptrap I've ever heard. Every single cancer and almost every single kind of treatment has a dose-response curve. Just because you can't measure it 
accurately or choose not to measure it doesn't make it go away. It's there. You can't ignore it. How many times do we use fractionated doses of radioactive iodine in the treatment of differentiated thyroid cancer? Give them a big dose, and you can cure them. Somehow we've forgotten that, and I think it plays into corporate interests to have 4 or 6 cycles of treatment whether or not these are needed.

Dr. Herrmann: You are a big supporter of the dual-tracer approach. Now we have the NETTER 2 study, for example, in higher-grade NETs. Do you think that in these kinds of patients a dual-tracer approach including ${ }^{18} \mathrm{~F}-\mathrm{FD}$ for patient selection is necessary?

Dr. Hicks: I personally think it's unethical not to do ${ }^{18} \mathrm{~F}-\mathrm{FDG}$ in patients with G3 tumors. The prevalence of ${ }^{18} \mathrm{~F}-\mathrm{FDG}$-avid non-somatostatin receptor-expressing tumor deposits is sufficiently high in that population that if you miss it, patients cannot benefit. That clone becomes the dominant clone very quickly, and the patients derive no benefit at all. Conversely, a short course of chemotherapy can eradicate these more aggressive clones and render the patient suitable for treatment with radioligand therapy. Again, this represents an example of the need for personalized therapy that involves appropriate sequencing of therapies rather than seeing them as competing treatment modalities.

Dr. Czernin: One of the most impressive achievements in nuclear medicine in Australia was the creation of the Australasian Radiopharmaceutical Trials Network. You were instrumental in starting this quite some time ago. Can you tell us a little bit about how that started and where it is going?

Dr. Hicks: Although Australia is a very big country geographically, it's a small country scientifically. The number of hospitals delivering specialized services is relatively small. We're also not a very mobile population. That makes follow-up less difficult, because patients don't change their primary care institutions or oncologists very often. We had the perfect storm, I think, in terms of a relatively immobile population being looked after in a few academic centers that all know each other, talk to each other, don't dislike each other, and aren't particularly competitive because we're in different cities a long way away apart, and patients tending not to travel anyway. So that was a real advantage and gave us the opportunity to do these sorts of collaborative trials. We also had (perhaps in the beginning more than now) a rather conducive regulatory environment within the public hospital system that allowed us to do investigator-driven trials.

Dr. Czernin: When did the network start?

Dr. Hicks: The forerunner of it was started in the late 1990s. We got together to be involved in the ${ }^{90}$ Y-DOTATOC trial that was run by Novartis in the late 1990 s, which was eventually published around 2010. We set up this network of people who were delivering or were interested in delivering PRRT in the late 1990s, and that was really the first cooperative trial. The broader network for clinical trials was subsequently driven by the Australasian Association of Nuclear Medicine Specialists. As you know, they have done both diagnostic and therapeutic trials in prostate cancer in recent times.

Dr. Herrmann: You told us that you are leaving Peter Mac in September 2022 after taking it from a very small shop to one of the most well-known sites worldwide. Everyone wants to know what is coming next. You're way too young to retire. What are you going to do next?

Dr. Hicks: I've learned a lot from Michael Phelps, David Kuhl, and, as noted, Markus Schwaiger, pioneers in our field. It's just been so special. At the same time, I've interacted with many industry groups involved in development of new paradigms. There is a mutual distrust between academia and industry that is not to the benefit of patients. A clear voice is needed to try to bring those things together for the benefit of patients. I'm planning to develop a company that will be focused on linking academia, industry, and government in a rational process to reframe the discussion around theranostics in such a way that it becomes patient-centered, not centered on the needs of industry, of the provider, and certainly not of government fiscal minimization, which is, in my mind, incredibly shortsighted. So many compelling economic arguments can be made for better health care. I figured that after 30 years, I've worked some of that out. What we practice at Peter Mac is a high level of patient-centered care. If I can bring that experience in a global sense, I think it will be beneficial for all 3 parties: industry, clinicians, and government.

Dr. Herrmann: This seems to be the perfect ending of this conversation. We wish you all the best in your future endeavors, and thank you for taking the time to talk to our readers. 